\title{
Mobile Robots Path Planning Based on Evolutionary Artificial Potential Fields Approach
}

\author{
Feilong Li \\ Faculty of computer and information science \\ Southwest University \\ Chongqing, China \\ lflstc@sina.cn \\ Yujun Wang \\ Faculty of computer and information science \\ Southwest University \\ Chongqing, China \\ wangyjun@swu.edu.cn
}

\author{
Yan Tan \\ Faculty of computer and information science \\ Southwest University \\ Chongqing, China \\ 478089780@qq.com \\ Gengyu Ge \\ Faculty of computer and information science \\ Southwest University \\ Chongqing, China \\ 948413217@qq.com
}

\begin{abstract}
This paper presents a new way for mobile robots' path planning which is based on the Evolutionary Artificial Potential Fields(EAPF) approach. The APF theory is a traditional method to plan path for a robot. The Evolutionary APF aims at helping a robot jump out of the local minimum point. Using a virtual goal to produce extra force and fixing the direction of the repulsive force are combined to prompt the robot to escape from the obstacle in different situations. The simulation result shows that the evolutionary method is effective for solving the local minimum problem.
\end{abstract}

Keywords-virtual goal; artifical potential fields; local minimum point; matrix; path planning

\section{INTRODUCTION}

Path planning is an important field of mobile robots, there are some major methods for path planning such as artificial potential fields(APF) approach, grid approach, neural network approach etc. Since the APF approach is so concise and efficient that it can be used for the real time path planning of mobile robots. However, the traditional APF has a serious defect, when the target is in the influence scope of obstacles, there are some other local minimum points, a robot may be trapped into the local minima, so that it can't reach the goal. There some kinds of methods aim at solving this problem, such as Local minima elimination [1], Tangent bug [2], Harmonic Potential [3] etc. Theses above only use a simple method but ignore the shape and size of obstacles. In this paper, two methods are used to avoid obstacles when the robot is trapped into a local minimum point.

Firstly, the evolutionary APF approach is used to drive the robot towards the goal. Secondly, two methods are combined to use for avoiding the obstacle when the robot is trapped. Thirdly, the evolutionary APF is reused to drive the robot move towards the real goal from the new starting point.

The simulation result is given out by MATLAB, and the result shows that using two methods combined is efficient and robust for path planning of mobile robots.

\section{EVOLUTIONARY ARTIFICALPOTENTIAL FIELDAS TEHORY AND LOCAL MiNIMUM PROBLEM}

\section{A. Traditional APF}

The APF [4-7] concept is first put forward by doctor Khatib in 1986, its main idea is defined as virtual potential fields for the robots. Obstacles are surrounded by repulsive potential filed which can produce repulsive force on robots, and the goal is surrounded by attractive potential filed which can produce attractive force on robots. The robot is driven towards the goal by the two kinds of forces.

The traditional attractive potential fields is defined in the literature [1] as below:

$$
U_{a t t}(X)=\frac{1}{2} k\left(X-X_{g}\right)^{2}
$$

where $X$ represents the position vector of a robot, $X_{g}$ represents the position vector of the goal, and $k$ is constant which represents the proportional coefficient of the attractive potential filed function. The attractive force is the negative gradient of this attractive potential fields function.

$$
F_{\text {att }}=-\operatorname{grad}_{\text {att }}(X)=-k\left(X-X_{g}\right)
$$

Then the repulsive potential fields function is defined as below:

$$
U_{\text {rep }}(X)= \begin{cases}\frac{1}{2} \eta\left(\frac{1}{\rho}-\frac{1}{\rho_{0}}\right)^{2} & \rho \leq \rho_{0} \\ 0 & \rho>\rho_{0}\end{cases}
$$

where $\eta$ represents the proportional coefficient of the repulsive potential filed function which is constant, and $\rho$ represents distance between the robot and the nearest point of the obstacle. $\rho_{0}$ is the scope of the obstacle can 
influence on robots. The repulsive force is the negative gradient of this repulsive potential fields function.

$$
F_{\text {rep }}=-\operatorname{grad}_{\text {rep }}(X)= \begin{cases}\eta\left(\frac{1}{\rho}-\frac{1}{\rho_{0}}\right) \frac{1}{\rho^{2}} \frac{\partial \rho}{\partial X} & \rho \leq \rho_{0} \\ 0 & \rho>\rho_{0}\end{cases}
$$

Then the composition of potential fields can be described by

$$
U(X)=U_{\text {rep }}(X)+U_{a t t}(X)
$$

The force on the robot is mainly caused by the potential fileds, and the composition of forces can be described by

$$
F(X)=F_{\text {rep }}(X)+F_{\text {att }}(X)
$$

The direction of the robot is depend on the force $F$.

The traditional APF approach is a kind of local path planning way. It can create virtual potential fields for the robot's motion quickly, but it may produce local minimum problem. When the robot, the obstacle and the goal are in the same line, the composition of forces on the robot may be equal to zero, then the robot will be trapped into the local minimum point.

\section{B. Evolutionary APF}

To solve the local minimum problem based on the traditional APF, some researchers have done some work. Literature [8] introduces a relative distance which considering the distance between the robot and the goal, this ensure that the target location is the globe minimum point, thus the robot can successfully reach the goal.

When the relative distance is added in the APF, the evolutionary repulsive potential fields function can be described by

$$
U_{\text {rep }}(X)= \begin{cases}\frac{1}{2} \eta\left(\frac{1}{\rho}-\frac{1}{\rho_{0}}\right)\left(X-X_{g}\right)^{n} & \rho \leq \rho_{0} \\ 0 & \rho>\rho_{0}\end{cases}
$$

where $\left(X-X_{g}\right)$ is the relative distance between the robot and the goal. The repulsive force is the negative gradient of repulsive potential fields function, and the composition of repulsive forces can be decomposed into two kinds of force(Figure 1).

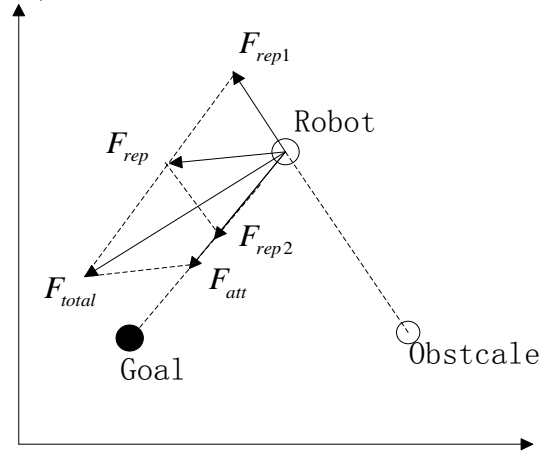

Figure 1. Force diagram of mobile robot

The composition of repulsive forces $F_{\text {rep }}$ can be described by

$$
F_{\text {rep }}= \begin{cases}F_{\text {rep } 1}+F_{\text {rep } 2} & \rho \leq \rho_{0} \\ 0 & \rho>\rho_{0}\end{cases}
$$

Both $F_{\text {rep } 1}$ and $F_{\text {rep } 2}$ are the decomposition of $F_{\text {rep }}, F_{\text {rep } 1}$ and $F_{\text {rep2 }}$ can be described as below:

$$
\begin{aligned}
& F_{\text {rep } 1}(X)=\eta\left(\frac{1}{\rho}-\frac{1}{\rho_{0}}\right) \frac{1}{\rho^{2}}\left(X-X_{g}\right)^{n} \\
& F_{\text {rep } 2}(X)=-\frac{n}{2} \eta\left(\frac{1}{\rho}-\frac{1}{\rho_{0}}\right)\left(X-X_{g}\right)^{n-1}
\end{aligned}
$$

The evolutionary APF can ensure the target location be the only zero point of potential fields. However, when the obstacle is in the line which connects the robot with goal, using this kind of evolutionary APF can't solve the local minimum effectively.

\section{LOCAL Minium POINT AVOIDANCE}

Two methods are introduced in different situations in this paper. Considering the efficient of the path planning and the size of obstacle, modifying the direction of repulsive force is adopted in common situation (Figure 2), establishing a virtual goal to attract the robot is adopted in the special situation (Figure 3).

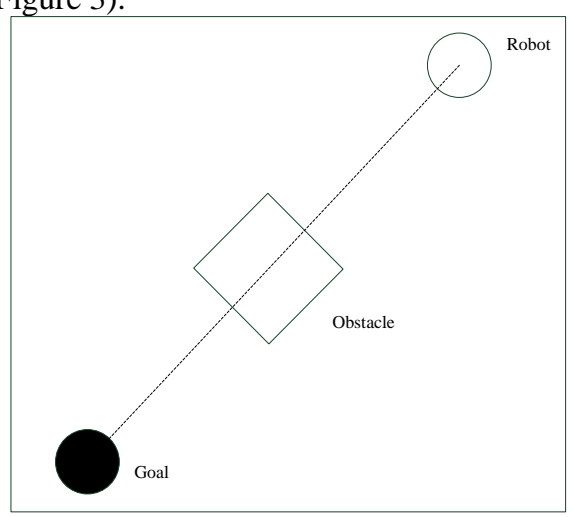

Figure 2. Common obstacle

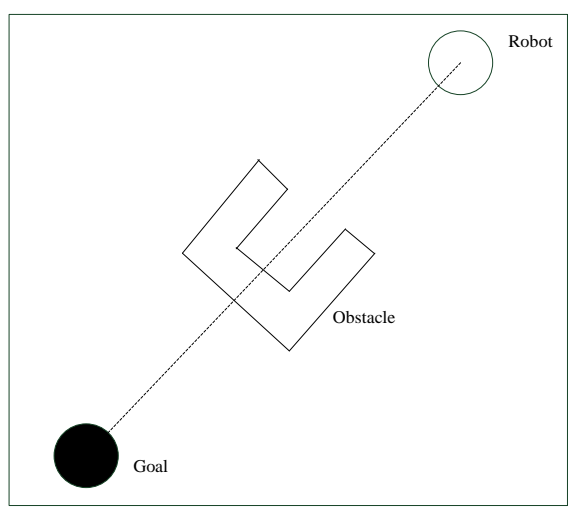

Figure 3. U-shaped obstacle 


\section{A. Modifying the Direction of Repulsive Force}

In the environment of Figure 2, when the robot is trapped into the local minimum point, modifying the direction of repulsive is an efficient way to help the robot to escape from local minimum. A is a matrix which is defined to modify the direction.

$$
A= \begin{cases}{\left[\begin{array}{cc}
\cos \alpha & -\sin \alpha \\
\sin \alpha & \cos \alpha
\end{array}\right]} & \theta=\pi \\
{\left[\begin{array}{ll}
1 & 0 \\
0 & 1
\end{array}\right]} & 0 \leq \theta \leq \frac{\pi}{2}\end{cases}
$$

In the matrix, $\alpha$ is a random value, and its value scope is

$$
0<\alpha \leq \frac{\pi}{2} \text {. }
$$

Because the robot will shake when the robot is trapped into the local minimum point, using the inequality below can judge whether the current point is in a local minimum point or not.

$$
\sqrt{\left(x_{i+1}-x_{i}\right)^{2}+\left(y_{i+1}-y_{i}\right)^{2}} \prec \varepsilon
$$

$\mathcal{E}$ is a infinitesimal. If the robot is trapped, the $F_{\text {rep }}$ will be modified by matrix A.

$$
F_{\text {nrep }}=A F_{\text {rep }}= \begin{cases}A F_{\text {rep } 1}+A F_{\text {rep } 2} & \rho \leq \rho_{0} \\ 0 & \rho>\rho_{0}\end{cases}
$$

The new repulsive force $F_{\text {nrep }}$ can repel the robot jump out of the local minimum point. The robot will move some steps until it avoids the obstacle, the evolutionary APF will be reused to drive the robot get closer to the goal.

\section{B. Establishing Virtual Goal}

In the environment of figure 3 , the obstacle is U-shaped, robots may not escape from the local minimum effectively using the first method mentioned above if it is trapped.

To help the trapped robot reach the goal, a virtual goal is set around the obstacle to produce an attractive force. Robot's sensor can detect the global information. First the sensor should measure the distance (d1, d2) between sides of obstacle and the robot. Then a virtual goal is set on the side of obstacle which the value of distance is smaller (Figure 4).

When the virtual goal is set, the real goal is ignored, the force on the robot can be described by

$$
F= \begin{cases}F_{\text {rep } 1}+F_{\text {rep } 2}+F_{\text {vatt }} & \rho \leq \rho_{0} \\ 0 & \rho>\rho_{0}\end{cases}
$$

where $F_{\text {vatt }}$ is a virtual attractive force produced by the virtual goal. The robot will be driven to reach the virtual goal, the evolutionary APF is reused to drive the robot get closer to the real goal. Besides the virtual goal will be ignored after the robot get to it.

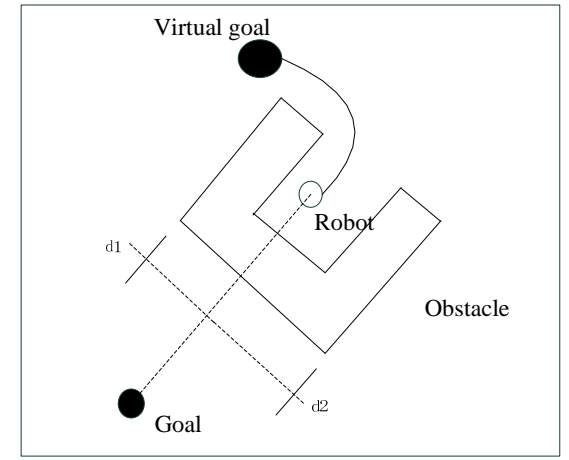

Figure 4. Setting virtual goal

\section{SimUlation ANALYSIS}

The evolutionary APF combined with two kinds of avoidance measures for path planning is test by MATLAB simulation experiments.

Figure 5 shows a path planed by the robot which is trapped into the local minimum point. The robot uses the first avoidance way to move away from the obstacle, and then get closer to the robot.

Figure 6 shows that a virtual goal is set to attract the robot to get out from the U-shaped obstacle when the robot is trapped.

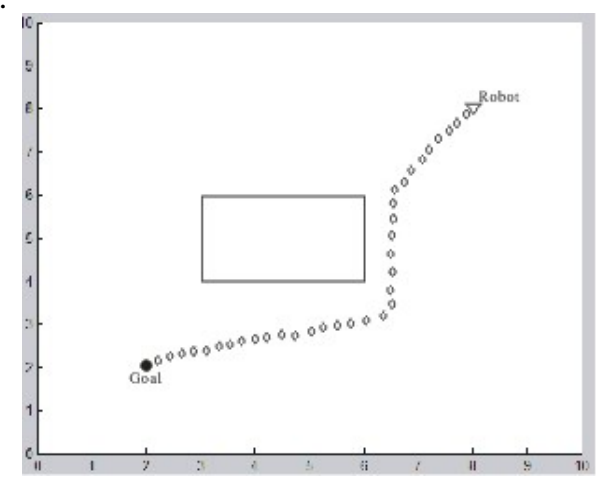

Figure 5. Common obstacle avoidance and pathe planning

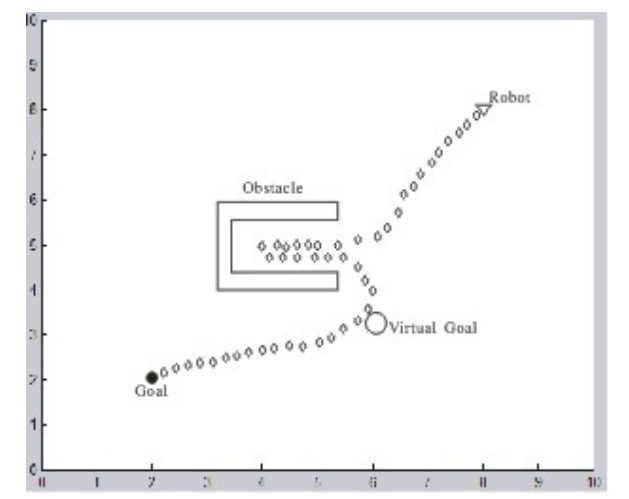

Figure 6. U-shaped obstacle avoidance and path planning

The simulation result shows that methods used in this paper is effective to solve the local minimum problem, but 
the angle of repulsive force which should be modified is random, we can't make sure that the path planning is optimal. Some other measures should be applied to improve the algorithm.

\section{ACKNOWLEDGMENT}

This paper focuses on solving the local minimum problem occurred in the path planning based on the evolutionary APF. Modifying the direction of repulsive force and establishing a virtual goal can make the robot jump out of the local minimum point and avoid the obstacle. The simulation result shows that the way used can solve the local minimum problem effectively.

\section{REFERENCES}

[1] Khatib. O, "Real-time Obstacle Avoidance for Manipulators and Mobile Robots," International Journal of Robotics Reseacher, vol. 5, no. 1 , pp. 90-98, 1986.

[2] Kamon. I, Rvin. E and Rimon. E, "A new range-sensor based globally convergent navigation algotithm for mobile robots," Proceedings of the.1996 IEEE International Conference on Robotics and Automation. Minneapolis,MN,USA:IEEE, vol. 1 , pp. 429-435, 1996.

[3] Jin-Oh. K and Pradeep. K, "Real-Time Obstacle Avoidance Using Harmonic Potential Functions," IEEE Trans on Robotics and Automation. vol. 3, no. 8, pp. 338-349, 1986.

[4] Z. Li, X. Chen and W. Xiao, "A New Motion Planning Approach Based on Artifical Potential Field In Unknown Environment," Lecture Notes in Computer Science. vol. 3320, pp. 376-382, 2004.

[5] Vadakkepat. P, Kay. C. T and Wang. M. L, "Evolutionary Artifical Potential Fields and their application in real time path palnning," Proceedings of the 2000 Congress on Evolutionary Compution. vol. 1, pp. 256-263, 2000.

[6] Zhang. J. Y and Liu. T, "Optimized Path Planning of Mobile Robot Based on Artifical Potential Filed," ACTA AERONAUTICA ET ASTRONAUTICA SINICA, vol. 18, pp. 183-188, 2007.

[7] Park. M. G and Lee. C. M, "Artifical Potential Filed Based Path Planning for Mobile Robots Using a Virtual Obstacle Concept," Advanced Intelligent Mechatronics, 2003. AIM 2003.Proceedings. 2003 IEEE International Conference, vol. 2, pp. 735 - 740, 2003.

[8] Wang. H. L, Fu. W. P and Fang. Z. D, "A path plan method for mobile robot based on improved potential field function," Machine Tool and Hydraulics, no. 6, pp. 67-68, 2002. 